WHAT ARE THE ECONOMIC IMPLICATIONS OF ANTITNF THERAPY IN PATIENTS WITH AXIAL SPONDYLOARTHRITIS? - RESULTS OF A HEALTH INSURANCE CLAIMS DATA ANALYSIS

I. Redeker ${ }^{1}$, F. Hoffmann ${ }^{2}$, J. Callhoff ${ }^{1}$, H. Haibel ${ }^{3}$, J. Sieper ${ }^{3}$, A. Zink ${ }^{1,4}$, D. Poddubnyy ${ }^{1,3} .{ }^{1}$ Epidemiology Unit, German Rheumatism Research Centre, Berlin; ${ }^{2}$ Department of Health Services Research, Carl von Ossietzky University, Oldenburg; ${ }^{3}$ Department of Gastroenterology, Infectiology and Rheumatology; ${ }^{4}$ Department of Rheumatology and Clinical Immunology, Charité Universitätsmedizin Berlin, Berlin, Germany

Background: Tumour necrosis factor (TNF)- $\alpha$ inhibitors are an effective but rather expensive treatment option in axial spondyloarthritis $(\mathrm{axSpA})$ patients who fail conventional treatment.

Objectives: The aim of this study was to analyse the changes in healthcare costs and resource utilisation after initiation of TNF- $\alpha$ inhibitors in patients with axSpA. Methods: Data of patients with axSpA newly exposed to TNF- $\alpha$ inhibitors between January 1, 2011 and December 31, 2015 were studied by using health insurance claims data in Germany. Resource utilisation, direct healthcare costs and productivity costs were assessed. Direct healthcare costs comprised costs for outpatient care (i.e., visits to physicians, laboratory visits, non-physician visits [e.g., visits to physical therapists], emergency department visits, outpatient hospital services, and aids [e.g., walkers]), costs for inpatient care (i.e., inpatient admissions) and costs for pharmacotherapy. Productivity costs comprised costs due to absence from paid work and were calculated using the friction cost method (FCM) and the human capital approach ( $\mathrm{HCA})$. Costs and resource utilisation were analysed the year before (baseline period) and the year after (follow-up period) initiation of TNF- $\alpha$ inhibitors.

Results: Data from 1452 persons were included in the analyses; mean age was 44.6 years and $47.9 \%$ were female. AxSpA-related pharmacotherapy use (nonsteroidal anti-inflammatory drugs [NSAIDs], non-opioid analgesics, opioid analgesics, systemic steroids, local steroids and conventional synthetic disease-modifying anti-rheumatic drugs [csDMARDs]) and admissions to hospital decreased significantly in the follow-up period compared to the baseline period. However, due to anti TNF- $\alpha$ therapy, mean direct healthcare costs increased from $€ 4494$ per patient in the baseline period to $€ 26473$ per patient in the follow-up period. Mean total costs increased from $€ 8072$ to $€ 29959$ using the HCA and from $€$ 6377 to $€ 28162$ using the FCM (Table). Excluding costs for anti-TNF therapy, total costs decreased by $15 \%$ to $€ 6876$ or by $20 \%$ to $€ 5080$ based on whether the HCA or the FCA was used.

Abstract SAT0287 - Table 1. Mean costs per patient before and after initiation of TNF- $\alpha$ inhibitors in axial spondyloarthritis $(n=1,452)$.

\begin{tabular}{|c|c|c|c|}
\hline & $\begin{array}{c}\text { Baseline } \\
\text { period } \\
\text { mean } \pm S D\end{array}$ & $\begin{array}{l}\text { Follow-up } \\
\text { period } \\
\text { mean } \pm S D\end{array}$ & P-value \\
\hline Outpatient care ${ }^{*}, €$ & $1,402 \pm 1,008$ & $1,381 \pm 1,054$ & 0.0285 \\
\hline Hospital admissions* ${ }^{*}, €$ & $1,890 \pm 3,681$ & $1,134 \pm 3,558$ & $<0.0001$ \\
\hline with diagnosis of axSpA* & $612 \pm 1582$ & $136 \pm 714$ & $<0.0001$ \\
\hline Pharmacotherapy, € & $1,201 \pm 2,939$ & $23,957 \pm 5,480$ & $<0.0001$ \\
\hline $\begin{array}{l}\text { excluding anti-TNF } \\
\text { therapy }\end{array}$ & $1,201 \pm 2,939$ & $875 \pm 1,721$ & $<0.0001$ \\
\hline NSAIDs & $130 \pm 159$ & $83 \pm 151$ & $<0.0001$ \\
\hline non-opioid analgesics & $21 \pm 77$ & $12 \pm 46$ & $<0.0001$ \\
\hline opioid analgesics & $118 \pm 517$ & $123 \pm 564$ & 0.3352 \\
\hline systemic steroids & $23 \pm 52$ & $17 \pm 54$ & $<0.0001$ \\
\hline local steroids & $1 \pm 6$ & $1 \pm 4$ & 0.0091 \\
\hline cSDMARDs & $159 \pm 366$ & $97 \pm 278$ & $<0.0001$ \\
\hline Absence at work, HCA, $€$ & $3,578 \pm 7,211$ & $3,486 \pm 7,896$ & 0.0458 \\
\hline Absence at work, FCM, $€$ & $1,883 \pm 2,943$ & $1,690 \pm 2,934$ & 0.0021 \\
\hline Total costs, $\mathrm{HCA}, €$ & $8,072 \pm 9,734$ & $29,959 \pm 10,679$ & $<0.0001$ \\
\hline $\begin{array}{l}\text { excluding anti-TNF } \\
\text { therapy }\end{array}$ & $8,072 \pm 9,734$ & $6,876 \pm 9,626$ & $<0.0001$ \\
\hline Total costs, FCM, € & $6,377 \pm 6,401$ & $28,162 \pm 7,609$ & $<0.0001$ \\
\hline $\begin{array}{l}\text { excluding anti-TNF } \\
\text { therapy }\end{array}$ & $6,377 \pm 6,401$ & $5,080 \pm 5,813$ & $<0.0001$ \\
\hline
\end{tabular}

Values are presented as mean \pm standard deviation. P-values were assessed using Wilcoxon signed-rank tests. Total costs per patient were calculated as the sum of the domains outpatient care, hospital admissions,

pharmacotherapy and absence at work.

TNF, Tumour Necrosis Factor; NSAIDs, Nonsteroidal Anti-Inflammatory Drugs; CSDMARDs, conventional synthetic Disease-Modifying Anti-Rheumatic Drugs; HCA, Human Capital Approach; FCM, Friction Cost Method; SD, standard deviation.

* excluding costs for pharmacotherapy.

Conclusions: Overall resource utilisation decreased after initiation of TNF- $\alpha$ inhibitors. The rise in pharmacotherapy costs driven by anti-TNF agents is accompanied by significantly lower costs for outpatient and inpatient care, as well as significantly lower productivity costs. However, the effect of TNF-blocker therapy on the patient's disease activity, function or quality of life could not be assessed in this analysis.

Acknowledgements: This work was supported by the Federal Ministry of Education and Research within the research network PROCLAIR (01EC1405).

Disclosure of Interest: None declared

DOI: 10.1136/annrheumdis-2018-eular.5075

\section{SAT0288 SECUKINUMAB DEMONSTRATES RAPID AND SUSTAINED EFFICACY IN ANKYLOSING SPONDYLITIS PATIENTS WITH NORMAL OR ELEVATED BASELINE CRP LEVELS: POOLED ANALYSIS OF TWO PHASE 3 STUDIES}

J. Braun $^{1}$, J. Sieper ${ }^{2}$, R. Landewé ${ }^{3}$, X. Baraliakos ${ }^{1}$, C. Miceli-Richard ${ }^{4}$, E. QuebeFehling $^{5}$, B. Porter ${ }^{6}$, K. Gandhi ${ }^{6}$, D. van der Heijde ${ }^{7} .{ }^{1}$ Rheumazentrum Ruhrgebiet Herne, Ruhr-University Bochum, Herne; ${ }^{2}$ Charité University Medicine Berlin, Berlin, Germany, ${ }^{3}$ University of Amsterdam and Atrium Medical Center, Amsterdam, Netherlands; ${ }^{4}$ Department of Rheumatology, Hôpital Bicêtre, Paris, France; ${ }^{5}$ Novartis Pharma AG, Basel, Switzerland; ${ }^{6}$ Novartis Pharmaceuticals Corporation, East Hanover, USA; ${ }^{7}$ Leiden University Medical Center, Leiden, Netherlands

Background: Elevated baseline (BL) CRP levels is one of the predictors of treat ment response in patients (pts) with active AS. ${ }^{1}$ The relationship between BL CRP levels and the treatment response to IL-17A inhibition has not been assessed in AS pts thus far.

Objectives: This post-hoc analysis assessed the response to secukinumab (SEC) treatment in AS pts with normal or elevated BL CRP from the phase 3 MEASURE 1 and MEASURE 2 studies over 3 years.

Methods: The study designs of MEASURE 1 and 2 have been reported. ${ }^{2}$ This analysis pooled data from all pts with available BL CRP levels who received subcutaneous (s.c.) SEC 150 mg (approved dose; $n=197$ ) or placebo (PBO; $n=195$ ). Efficacy endpoints included ASAS20/40, BASDAI, BASDAI50, ASDAS inactive disease, and ASAS partial remission (PR) stratified by normal $(<5 \mathrm{mg} / \mathrm{L})$ and ele vated ( $\geq 5 \mathrm{mg} / \mathrm{L}$ ) BL CRP; elevated BL CRP group was further subdivided into a range of elevated CRP levels: $>5-10,>10-15,>15 \mathrm{mg} / \mathrm{L}$. Data are presented as non-responder imputation (NRI) at Week (Wk)16 and multiple imputation (MI) a Wk156 for binary variables and mixed-effect model repeated measure (MMRM) at Wks 16 and 156 for continuous variables.

Results: Overall, 36.5\% (143/392) of pts with normal CRP and 63.5\% (249/392) of pts with elevated BL CRP were included in the pooled analysis. BL characteristics were balanced across normal and elevated BL CRP groups. ${ }^{3}$ At Wk16, efficacy endpoints were improved with SEC $150 \mathrm{mg}$ vs PBO in pts with normal or elevated BL CRP. ${ }^{3}$ Results were consistent across all levels of elevated BL CRP with a trend for greater improvement in pts with more elevated CRP (Table). Improvements were sustained or further improved at Wk156 in all groups (Table).

Abstract SAT0288 - Table 1. Summary of Clinical Efficacy

\begin{tabular}{|c|c|c|c|c|c|c|c|c|c|}
\hline \multirow[b]{3}{*}{ Clinical outcomes } & \multirow[b]{3}{*}{ Wk } & \multirow{2}{*}{\multicolumn{2}{|c|}{$\begin{array}{c}\text { Normal BL CRP } \\
(<5 \mathrm{mg} / \mathrm{L})\end{array}$}} & \multicolumn{6}{|c|}{ Elevated BL CRP } \\
\hline & & & & \multicolumn{2}{|c|}{$>5-10 \mathrm{mg} / \mathrm{L}$} & \multicolumn{2}{|c|}{$>10-15 \mathrm{mg} / \mathrm{L}$} & \multicolumn{2}{|c|}{$>15 \mathrm{mg} / \mathrm{L}$} \\
\hline & & $\begin{array}{c}\mathrm{SEC} \\
(\mathrm{N}=72)\end{array}$ & $\begin{array}{c}\mathrm{PBO} \\
(\mathrm{N}=71)\end{array}$ & $\begin{array}{c}\mathrm{SEC} \\
(\mathrm{N}=38)\end{array}$ & $\begin{array}{l}\mathrm{PBO} \\
(\mathrm{N}=31)\end{array}$ & $\begin{array}{c}\mathrm{SEC} \\
(\mathrm{N}=19)\end{array}$ & $\begin{array}{c}\text { PBO } \\
(\mathrm{N}=31)\end{array}$ & \begin{tabular}{c|}
$\mathrm{SEC}$ \\
$(\mathrm{N}=68)$
\end{tabular} & $\begin{array}{c}\mathrm{PBO} \\
(\mathrm{N}=62)\end{array}$ \\
\hline \multirow{2}{*}{ ASAS20, $\%$} & 16 & $56.9^{5}$ & 28.2 & 42.1 & 32.3 & $68.4^{\S}$ & 29.0 & $73.5^{*}$ & 27.4 \\
\hline & 156 & 61.3 & - & 65.0 & - & 82.8 & - & 85.0 & - \\
\hline \multirow{2}{*}{ ASAS40, $\%$} & 16 & $34.7^{\top}$ & 7.0 & 31.6 & 16.1 & 42.1 & 22.6 & $48.5^{*}$ & 11.3 \\
\hline & 156 & 42.4 & - & 50.0 & - & 76.4 & - & 67.9 & - \\
\hline \multirow{2}{*}{$\begin{array}{l}\text { BASDAl, LS mean } \\
\text { change from BL }\end{array}$} & 16 & $-2.2^{\dagger}$ & -1.0 & -1.5 & -0.7 & $-2.8^{\dagger}$ & -0.6 & $-2.8^{*}$ & -0.6 \\
\hline & 156 & -2.7 & - & -2.8 & - & -3.6 & - & -3.7 & - \\
\hline \multirow{2}{*}{ BASDAI50, \% } & 16 & $27.8^{5}$ & 7.0 & 34.2 & 19.4 & $42.1^{5}$ & 6.5 & $41.2^{*}$ & 8.1 \\
\hline & 156 & 40.4 & - & 56.1 & - & 75.5 & - & 58.5 & - \\
\hline \multirow{2}{*}{$\begin{array}{l}\text { ASDAS } \\
\text { inactive disease, } \\
\%\end{array}$} & 16 & $19.4^{7}$ & 4.2 & 21.1 & 6.5 & 26.3 & 0 & 8.8 & 1.6 \\
\hline & 156 & 28.9 & - & 28.5 & - & 16.9 & - & 15.1 & - \\
\hline \multirow{2}{*}{ ASAS PR, \% } & 16 & 12.5 & 2.8 & $23.7^{2}$ & 3.2 & 15.8 & 3.2 & 11.8 & 4.8 \\
\hline & 156 & 14.4 & - & 34.6 & - & 33.4 & - & 30.2 & - \\
\hline
\end{tabular}

Conclusions: SEC $150 \mathrm{mg}$ demonstrated sustained efficacy through 3 years in AS patients with both normal and elevated CRP levels, with an increased effect in elevated CRP patients.

REFERENCES:

[1] Davis JC Jr, et al. J Rheumatol. 2005;32:1751-4.

[2] Baeten D, et al. N Engl J Med 2015;373:2534-48.

[3] et al. Arthritis Rheumatol 2017;69

[4] Braun J, et al. Arthritis Rheumatol 2017;69(suppl 10).

Acknowledgements: The study was sponsored by Novartis Pharma AG 